\title{
Study of Nonlinear Dynamics of LDPC Decoders
}

\author{
Xia Zheng* \\ Francis C.M. Lau*
}

\begin{abstract}
Low-density-parity-check (LDPC) codes have aroused much research interest because of their excellent bit error performance. The behaviour of the iterative LDPC decoders, however, has not been fully investigated at all signal-to-noise ratios (SNRs). By considering the LDPC decoders as high-dimensional nonlinear dynamical systems, we attempt to study the corresponding phase trajectories at different SNR values. By having an in-depth understanding of the decoder behaviour, engineers should be able to design more effective and efficient decoders.
\end{abstract}

\section{INTRODUCTION}

Both turbo codes [1]-[3] and low-density-paritycheck (LDPC) codes [4] have attracted much research attention in the last decade because they can provide bit error performance close to the limit derived by Shannon. To decode turbo and LDPC codewords, iterative decoding algorithms have been proposed. While some researchers focus on the constructions of the "good" codes and low-complexity encoding/decoding techniques, others are interested in studying the behaviour of the iterative decoding algorithms.

In the pioneering papers $[1,2]$, Richardson, Agrawal and Vardy studied in detail the performance and nature of the turbo decoding algorithm by modelling it as a discrete dynamical system. They showed that in addition to fixed points, bifurcations leading to period doubling and oscillations may be produced by the decoding algorithm. Later, Kocarev et al. [3] also found that chaos existed in turbo decoding and a control method was proposed to improve the convergence rate under such a scenario. As for the LDPC decoding, the convergence behaviour of the bit error rate (BER) has been investigated using Gaussian approximation [4]. Bifurcation has been found as the SNR varies. However, the analyses only provide us with an asymptotical performance as the block length tends to infinity. For codes with finite length, the performance and behaviour of the decoder have not been fully evaluated.

In this paper, we attempt to study the behaviour of finite-length LDPC decoders in depth by analyzing the phase trajectories of the posterior probabil-

\footnotetext{
*Department of Electronic Information and Engineering, Hong Kong Polytechnic University, Hong Kong. Emails: \{xia.zheng,encmlau,encktse,enscwong\}@polyu.edu.hk. This work was supported in part by a research grant provided by Hong Kong Polytechnic University.
}

\author{
Chi K. Tse* Siu Chung Wong*
}

ities. Extensive simulations have been performed for a wide range of signal-to-noise ratios (SNRs). In the next section, we will briefly review some notations of the LDPC codes and the iterative decoding process. Simulation results will then be presented and discussed in Sect. 3.

\section{LDPC CODES AND THE DECODING ALGORITHM}

Low-density-parity-check codes are linear block codes and all linear block codes can be represented by bipartite graphs consisting of two sets of nodes, namely variable nodes and check nodes. The key property of LDPC codes is the sparsity of the graph. In other words, the degree of all nodes is low. Also, there are two kinds of LDPC codes: regular and irregular. For regular LDPC codes, all nodes of the same type have the same degree. For irregular LDPC codes, the degree of each set of nodes is chosen according to some distributions. For a given distribution pair $(\lambda, \rho)$ of a LDPC ensemble,

$$
\lambda(x):=\sum_{i=2}^{d_{v}} \lambda_{i} x^{i-1} \quad\left(\rho(x):=\sum_{i=2}^{d_{c}} \rho_{i} x^{i-1}\right)
$$

specifies the variable (check) node degree distribution where $d_{v}$ and $d_{c}$ denote the maximum variable degree and check degree, respectively.

Consider a binary-input additive white Gaussian noise channel and a transmitted codeword with a block length $n$. Denote the $i$ th code bit $(i=1,2, \ldots, n)$ by $c_{i} \in\{0,1\}$. The transmitted signal corresponding to this code bit is denoted by $s_{i} \in\{-1,1\}$ and equals $(-1)^{c_{i}}$. The received signal, denoted by $y_{i}$, is given by $y_{i}=s_{i}+z_{i}$, where the variables $z_{i}$ are independent and identically distributed zero-mean Gaussian random variables with variance (noise power) $\sigma^{2}$.

Suppose a message-passing algorithm is used in the iterative decoder to decode the LDPC codes. Define $q_{i j}(b)(b \in\{0,1\})$ as the probability computed based on (i) the received signal $y_{i}$, and (ii) the message $r_{j^{\prime} i}(b)$ passed from the neighbouring check nodes $C_{i}$ excluding the check node $j$. Also, we define $r_{j i}(b)$ as the probability computed based on the message $q_{i^{\prime} j}(b)$ passed from the neighbouring variable nodes $V_{j}$ excluding the variable node $i$. 
The message-passing algorithm in the probability domain then proceeds as follows.

1. Estimate the noise power $\sigma^{2}$. Then for $i=$ $1,2, \ldots, n$, initialize $P_{i}(b)=\operatorname{Prob}\left(c_{i}=b \mid y_{i}\right)$. Set $q_{i j}(b)=P_{i}(b)$ if the variable node $i$ and the check node $j$ are connected.

2. Update $r_{j i}(b)$ using

$$
\left\{\begin{array}{l}
r_{j i}(0)=\frac{1}{2}+\frac{1}{2} \prod_{i^{\prime} \in V_{j} / i}\left(1-2 q_{i^{\prime} j}(1)\right) \\
r_{j i}(1)=1-r_{j i}(0) .
\end{array}\right.
$$

3. Update $q_{i j}(b)$ using

$$
q_{i j}(b)=K_{i j} P_{i}(b) \prod_{j^{\prime} \in C_{i} / j} r_{j^{\prime} i}(b) \quad b=0,1
$$

where $K_{i j}$ is chosen to meet $q_{i j}(0)+q_{i j}(1)=1$.

4. Compute the posterior probability of the code bit $c_{i}$ using

$$
Q_{i}(b)=K_{i} P_{i}(b) \prod_{j \in C_{i}} r_{j i}(b) \quad b=0,1
$$

where $K_{i}$ is chosen to ensure that $Q_{i}(0)+$ $Q_{i}(1)=1$.

5. Set $Q_{i}(1) \underset{\hat{c}_{i}=0}{\stackrel{\hat{c}_{i}=1}{\gtrless}} Q_{i}(0)$. If $\hat{\boldsymbol{c}} \boldsymbol{H}^{T}=0$ or the number of iterations equals the maximum limit, stop; else, go to Step 2. Here, $\boldsymbol{H}$ is the parity check matrix of the LDPC codes.

The whole iterative process can also be written as

$$
\begin{cases}\boldsymbol{q}^{l}(b, \sigma)=f_{1}\left(\boldsymbol{r}^{l}(b, \sigma)\right) & b=0,1 \\ \boldsymbol{r}^{l+1}(b, \sigma)=f_{2}\left(\boldsymbol{q}^{l}(b, \sigma)\right) & b=0,1\end{cases}
$$

where $l=0,1,2, \ldots$ denotes the iteration number, $\boldsymbol{r}^{l}(b, \sigma)$ and $\boldsymbol{q}^{l}(b, \sigma)$ are both vectors of length $n / \int_{0}^{1} \lambda(x) d x$ parameterized by $\sigma$.

It can be observed that the whole process is parameterized by the probabilities $\operatorname{Prob}\left(c_{i}=b \mid y_{i}\right)$ $(i=1,2, \ldots, n)$, which are determined by the transmitted codeword and the noise values. Simulations have also shown that the iterative decoder is very sensitive to such parameters.

Suppose that a fixed point exists in the dynamical system. The stability of the fixed point can be determined from the eigenvalues of the Jacobians of the iterative system of (4) evaluated at the fixed point. It is well-known that if all the eigenvalues lie in the unit circle, the fixed point is stable. If an eigenvalue approaches 1 or -1 , flip bifurcation or fold bifurcation would occur, respectively. Also, Neimark-Sacker bifurcation occurs when a pair of complex conjugate eigenvalues move towards the unit circle from inside.

Although the entire phase trajectories of $\boldsymbol{r}^{l}(b, \sigma)$ and $\boldsymbol{q}^{l}(b, \sigma)$ can be found, it is not practical to plot and study them because both $\boldsymbol{r}^{l}(b, \sigma)$ and $\boldsymbol{q}^{l}(b, \sigma)$ are very high dimensional variables in the order of tens of thousands. Instead, we make use of the measure $\mathrm{E}(l)$ to investigate the dynamical behaviour of the decoder, where $\mathrm{E}(l)$ is defined as the mean square value of the posterior probabilities of the code bits being equal to 0 at the $l$ th iteration, i.e.,

$$
\mathrm{E}(l)=\frac{1}{n} \sum_{i=1}^{n}\left[Q_{i}^{l}(0)\right]^{2} .
$$

In our study, codewords with all zeros are used because it is known that the all-zero codewords are adequate for assessing the performance of a linear code with a symmetrical channel and a symmetrical decoding algorithm. Therefore, if all code bits are detected correctly after some iteration number $l, Q_{i}^{l}(0)=1$ for all $i$ and $\mathrm{E}(l)=1$.

\section{RESULTS AND DISCUSSION}

Suppose that the noise samples are represented by $\boldsymbol{z}=\left(z_{1}, z_{2}, \ldots, z_{n}\right)$. If the ratios between consecutive sample values, i.e., $z_{1} / z_{2}, z_{2} / z_{3}, \ldots, z_{n-1} / z_{n}$ are fixed, we denote such noise samples as one noise realization. Different noise realizations correspond to different noise-ratios vectors. For a given noise realization, the noise vector $\boldsymbol{z}$ is completely determined by the signal-to-noise ratio (SNR) because $1 /(2 R \times \mathrm{SNR})=\hat{\sigma}^{2}=\frac{1}{n} \sum_{i=0}^{n-1} z_{i}^{2}$ where $R$ is the code rate. Usually, $n$ is a large integer and $\hat{\sigma}^{2}$ will be a good approximation of the channel noise variance $\sigma^{2}$.

Extensive simulations have been performed. It is observed that decoders for regular LDPC codes have similar behaviour as those for irregular codes. In particular, fold bifurcation, flip bifurcation and Neimark-Sacker bifurcation occur within a certain range of SNR called the "waterfall region". Due to the shortage of space, we will only discuss the results for the irregular LDPC codes here. We consider an irregular $(1008,504)$ LDPC code and study the trajectories of the iterative decoding algorithm.

For some noise realizations, only fold bifurcations occur as we change the SNR. Fig. 1 shows the changes in the phase trajectories induced by a typical fold bifurcation. The figures on the left hand side plot the value of $\mathrm{E}(l)$ against $l$ whereas those on the right plot the number of error bits against $l$. At an SNR value of $0.91178510 \mathrm{~dB}$, the phase trajectory of the LDPC decoder converges to a stable indecisive fixed point, as indicated by the 

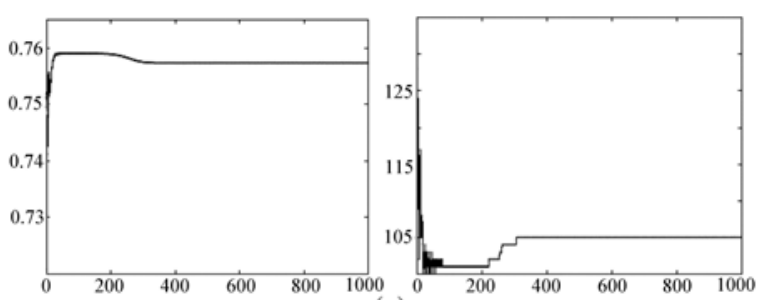

(a)
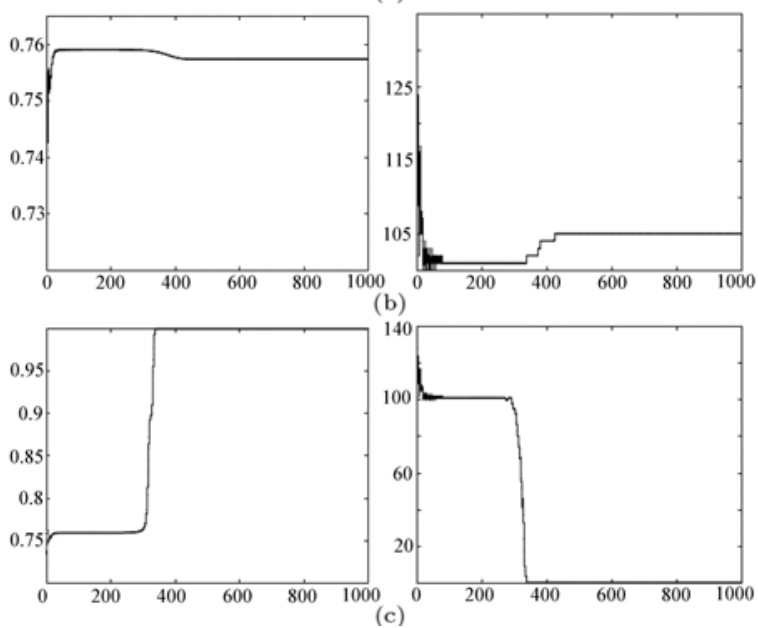

(b)

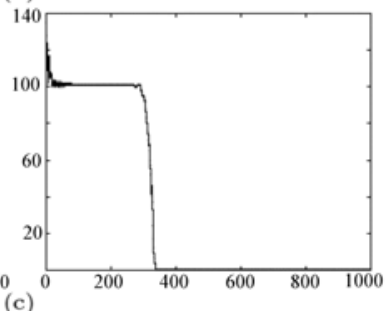

Figure 1: A typical fold bifurcation. Left: $\mathrm{E}(l)$ versus $l$. Right: Number of error bits versus $l$. (a) $\mathrm{SNR}=0.91178510 \mathrm{~dB}$; (b) $\mathrm{SNR}=0.91178518 \mathrm{~dB}$; (c) $\mathrm{SNR}=0.91178520 \mathrm{~dB}$.

plots. As the SNR is increased to $0.91178518 \mathrm{~dB}$, the fixed point becomes less stable and the decoder takes a longer time to converge. When the SNR is further increased to $0.91178520 \mathrm{~dB}$, the indecisive fixed point disappears and the phase trajectory is able to move away from this neighbourhood and converge to an unequivocal fixed point. In Fig. 2, the values of $\mathrm{E}(l)$ at the steady state is plotted against SNR. It can be seen that there are two distinct SNR regions separated by fold bifurcation.

For some other noise realizations, both flip bifurcations and Neimark-Sacker bifurcations occur. Fig. 3 illustrates such bifurcations occurring at the LDPC decoder. As expected, at low SNR $(0.3 \mathrm{~dB})$, the LDPC decoding algorithm converges to a stable indecisive fixed point. When the SNR increases, the indecisive fixed point becomes less stable. At an SNR of $0.55 \mathrm{~dB}$, the fixed point undergoes a flip bifurcation and a stable period-two cycle is formed. The periodic cycle starts to lose its stability at SNR $=0.592 \mathrm{~dB}$. Eventually, the system bifurcates at $\mathrm{SNR}=0.601 \mathrm{~dB}$ and once again, the trajectory converges to an indecisive fixed point. At $\mathrm{SNR}=0.615 \mathrm{~dB}$, the phase trajectory oscillates with diminishing amplitude and it takes longer to converge. At SNR $=0.65 \mathrm{~dB}$, the fixed

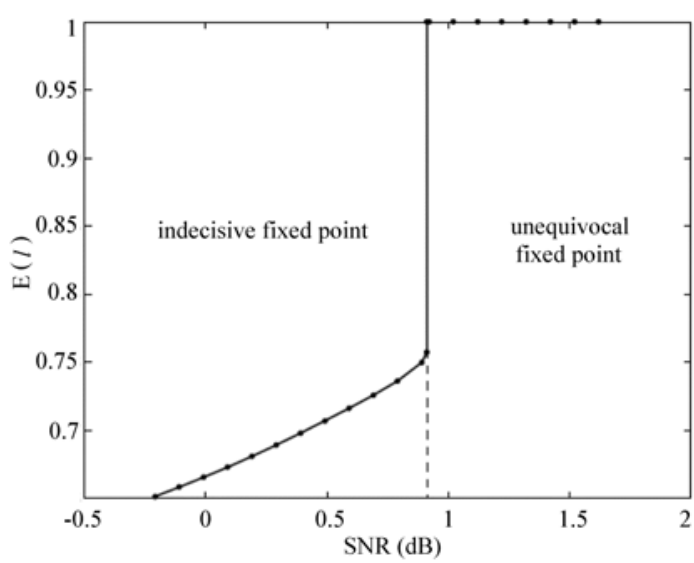

Figure 2: Steady-state $\mathrm{E}(l)$ versus SNR. Fold bifurcation occurs. The last 50 points of $\mathrm{E}(l)$ at each SNR are plotted.

point has undergone Neimark-Sacker bifurcation, through which the phase trajectory goes into an invariant set and as a result, after a transient period, the phase trajectory becomes quasi-periodic. As SNR increases further, the trajectory finally loses its stability at SNR $=0.90 \mathrm{~dB}$ and chaos occurs. At an SNR of $1.03 \mathrm{~dB}$, the LDPC decoding algorithm converges to an unequivocal fixed point.

In Fig. 4, the values of $\mathrm{E}(l)$ at the steady state are plotted against SNR again. It can be observed that bifurcations occur in the SNR range of around $0.45 \mathrm{~dB}$ to $1 \mathrm{~dB}$. Comparing Fig. 2 and Fig. 4, we also find that although different noise realizations produce different bifurcation diagrams, the whole SNR range in all cases can be divided into three regions, namely low-SNR region corresponding to indecisive fixed points; waterfall region where bifurcations occur; and high-SNR region corresponding to unequivocal fixed points.

\section{CONCLUSION}

In this paper, we have studied the behaviour of LDPC decoders for irregular codes in detail. Simulation results have shown that bifurcations, including fold, flip and NS bifurcations, occur at the decoders at the "waterfall" SNR region. In the "waterfall" region, oscillations and chaos are produced and the decoding algorithms do not converge. However, the waterfall region varies for different noise realizations. Our next task is thus to identify the exact "waterfall" region. We will then propose and investigate decoding algorithms with an aim to removing chaos and improving the convergence rate in the "waterfall" region. The final goal is to optimize the overall performance of LDPC decoders in terms of complexity, speed and error rate. 


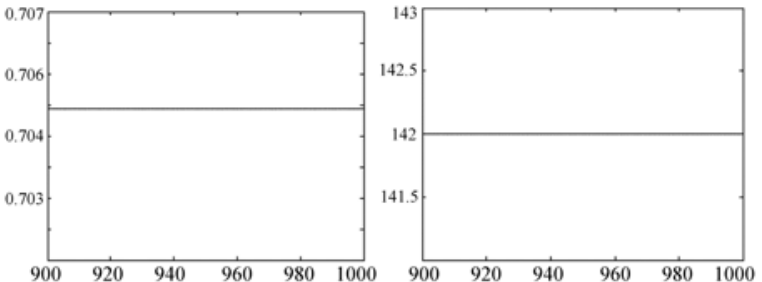

(a)
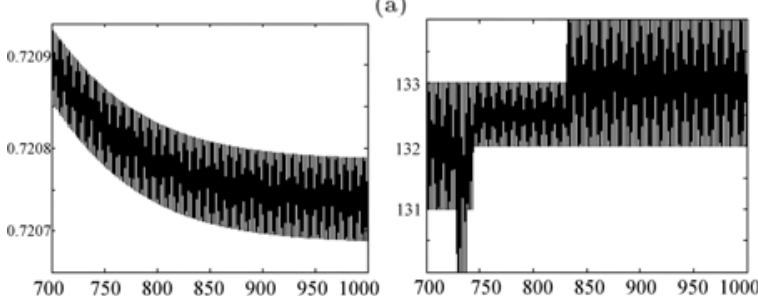

(b)
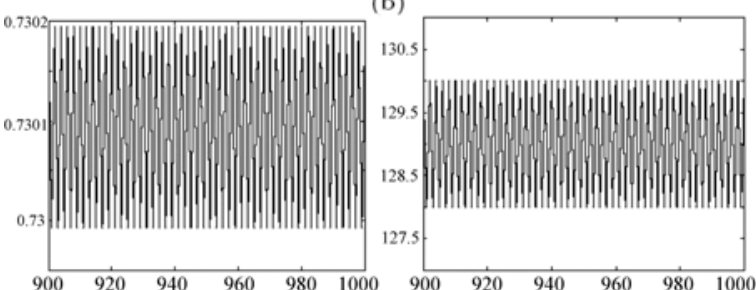

(c)

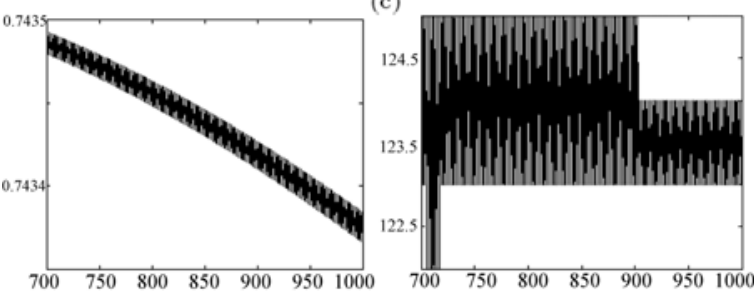

(d)
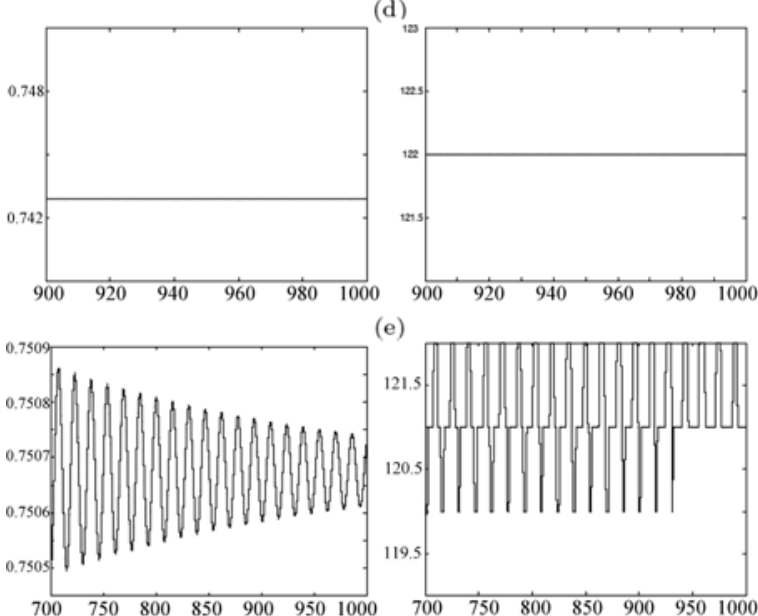

(e)
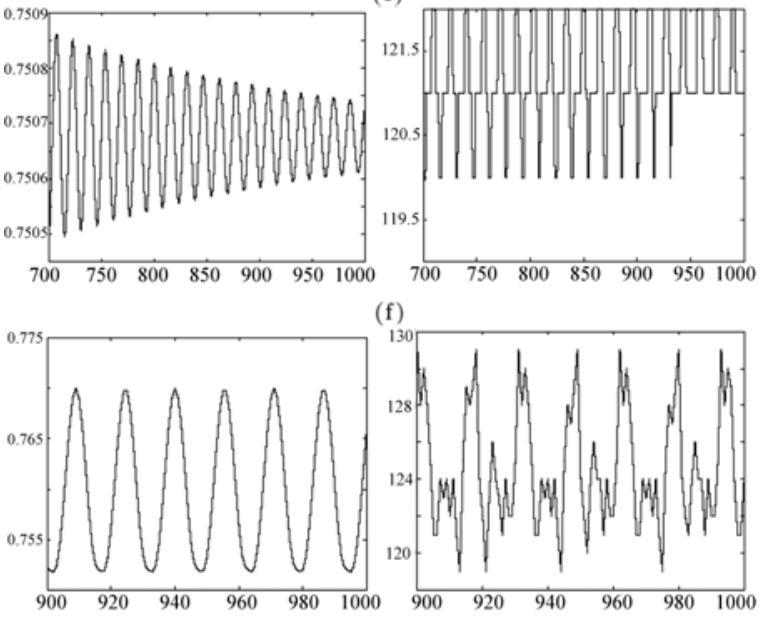

(g)
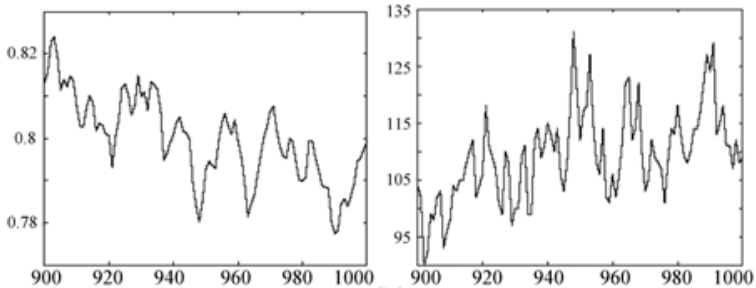

(h) 900
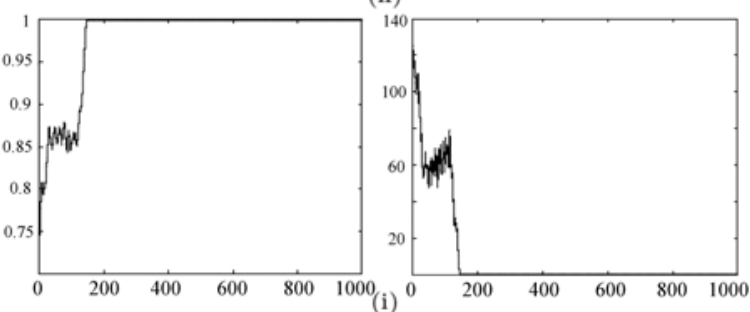

Figure 3: Occurrences of flip and Neimark-Sacker bifurcations. Left: $\mathrm{E}(l)$ versus $l$. Right: Number of error bits versus $l$. Value of SNR: (a) $0.30 \mathrm{~dB}$; (b) $0.47 \mathrm{~dB}$; (c) $0.55 \mathrm{~dB}$; (d) $0.592 \mathrm{~dB}$; (e) $0.601 \mathrm{~dB}$; (f) $0.615 \mathrm{~dB}$; (g) $0.65 \mathrm{~dB}$; (h) $0.90 \mathrm{~dB}$; (i) $1.03 \mathrm{~dB}$.

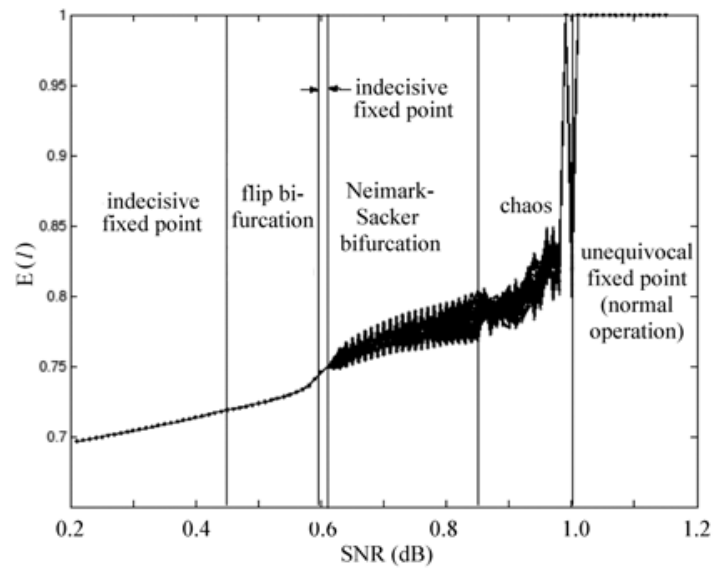

Figure 4: Steady-state $\mathrm{E}(l)$ versus SNR. Flip and NS bifurcations occur. The last 50 points of $\mathrm{E}(l)$ at each SNR are plotted.

\section{References}

[1] T. Richardson, "The geometry of turbo-decoding dynamics," IEEE. Trans. Inform. Theory, vol. 46, pp. 9-23, Jan. 2000.

[2] D. Agrawal and A. Vardy, "The turbo decoding algorithm and its phase trajectories," IEEE. Trans. Inform. Theory, vol. 47, pp. 699-722, 2001.

[3] L. Kocarev, Z. Tasev and A. Vardy, "Improving turbo codes by control of transient chaos in turbodecoding algorithms," Electronics Letters, vol. 38, pp. 1184-6, Sept. 2002.

[4] S.Y. Chung, T.J. Richardson and R. Urbanke, "Analysis of sum-product decoding of low-density parity-check codes using a Gaussian approximation," IEEE. Trans. Inform. Theory, vol. 47, pp. 657-670, Feb. 2001. 